\title{
Forecast of Total Energy Consumption in Shandong Province Based on Grey BP Model
}

\author{
Mengyao Mei ${ }^{1,}$, , Lili $\mathrm{Ma}^{2}$, Zhihong Liu ${ }^{3}$, Zhongxian Zhu ${ }^{3}$, Jianan Li ${ }^{1}$, Xiaohan Fang ${ }^{3}$ \\ ${ }^{1}$ Department of Electrical Engineering, Rongcheng College, Harbin University of Science and Technology, Rongcheng, China \\ ${ }^{2}$ Department of Economic Management, Rongcheng Campus of Harbin University of Science and Technology, Rongcheng, China \\ ${ }^{3}$ Software Engineering Department, Rongcheng College, Harbin University of Science and Technology, Rongcheng, China
}

Email address:

1542574006@qq.com (Mengyao Mei),961566805@qq.com (Lili Ma)

*Corresponding author

\section{To cite this article:}

Mengyao Mei, Lili Ma, Zhihong Liu, Zhongxian Zhu, Jianan Li, Xiaohan Fang. Forecast of Total Energy Consumption in Shandong Province Based on Grey BP Model. International Journal of Energy and Power Engineering. Vol. 7, No. 3, 2018, pp. 40-46. doi: 10.11648/j.ijepe.20180703.13

Received: October 8, 2018; Accepted: November 5, 2018; Published: November 8, 2018

\begin{abstract}
With the continuous increase of China's total energy consumption, we can find the rule and grasp its development trend from the change trend of energy consumption. In order to provide scientific basis for rational use of energy. In this paper,firstly, based on the data of total energy consumption of shandong province from 2007 to 2016, grey prediction model and $\mathrm{BP}$ neural network were used to predict total energy consumption of shandong province from 2007 to 2016. MATLAB was used to calculate the predicted value of each year and the average relative error of the two models was $7.25 \%$ and $3.70 \%$ respectively. Secondly, on the basis of the grey prediction model, BP neural network was used to correct the predicted value of total energy in shandong province. Then, the grey BP modified model was used to obtain the total energy consumption of shandong province from 2007 to 2016. MATLAB was used to calculate the predicted values of each year and the average relative error of the modified model was $2.04 \%$. Finally, the total energy consumption of shandong province in 2018-2035 is predicted. The results show that the average relative error is small and the prediction effect is obvious. This shows that the grey BP model is effective in predicting total energy consumption.
\end{abstract}

Keywords: Grey Prediction, BP Neural Network, Grey BP Model, Total Energy Consumption

\section{Introduction}

The production and use of energy is an important part of the national economy. Predicting and analyzing the total energy consumption is of vital importance for exploring the law of energy consumption and how to save energy. Through the analysis of China's total energy consumption over the years, it is found that China's total energy consumption shows an upward trend. According to the relevant data, the commonly used methods in the prediction of total energy consumption include LMDI method, multiple regression trial analysis model, gray prediction method and BP neural network.

Zhang Ming, Mu Hailin scholars constructed a decomposition model of passenger transportation energy consumption in China's megacities based on LMDI method, and applied the model to Shanghai, Beijing and other places [1]. Dai Xiaowen, He Yanqiu decomposed the factors affecting agricultural carbon emissions based on Kaya's identity, and then used the LMDI index decomposition method to analyze the driving strength and contribution rate of these factors [2]. It is concluded that the improvement of rural living standards is the most main factor in promoting agricultural carbon emissions [3]. Liu Yuhai and Wu Peng used the SBM-Undesirable model and the Meta-frontier production function as a comprehensive analysis framework to measure the economic growth efficiency of 17 members of the APEC region during the period of 1980-2007 under the dual constraints of energy consumption and carbon dioxide emissions [4]. The measurement factors of economic growth 
efficiency under double constraints are tested [5].

In this paper, the gray prediction model is used to make a preliminary prediction of the total energy consumption in Shandong Province. Secondly, the BP neural network is used to make predictions again. Then the gray BP model is used for correction. Finally, the total energy consumption of Shandong Province is predicted from 2018 to 2025 . Therefore, it is verified that the gray BP model is more effective in the analysis of the total energy consumption.

\section{Method Basis}

\subsection{Grey Forecast Model}

The grey prediction model is a model that establishes relationships based on certain information and predicts uncertain information. The model mainly uses the accumulative method to initialize the data to generate a data sequence with strong regularity.

a) Gray sequence data processing - initial value processing Suppose the original data is listed below

$$
x^{(0)}=\left\{x^{(0)}(i)\right\}, x^{(0)}(i) \geq 0, i=1,2 \cdots n
$$

After initializing $x^{(0)}, x^{(1)}$ is obtained, then:

$$
x^{(1)}(i)=\left\{\frac{x^{(0)}(i)}{x^{(0)}(1)}\right\}, i=1,2, \cdots, n
$$

b) Gray sequence generation method

Suppose the original data is $\left\{x^{(0)}(i)\right\}$, and $x^{(0)}(i) \geq 0, i=1,2 \cdots n$, If $x^{(1)}(i)$ and $\left\{x^{(0)}(i)\right\}$ satisfy the following relationship, namely:

$$
x^{(0)}(k)=\sum_{m-1}^{k} x^{(0)}(m)
$$

Among them, the r-time cumulative generation sequence has the following relationship:

$$
x^{(r)}(k)=x^{(r)}(k-1)+x^{(r-1)}(k)
$$

c) Grey differential equation

Assume that the differential equation is:

$$
\frac{d x}{d t}+a x=b
$$

Discretize the variable to get:

$$
\frac{d x}{d t}=\frac{x^{(1)}(k)-x^{(1)}(k-1)}{k-(k-1)}=x^{(1)}(k)-x^{(1)}(k-1)=x^{(0)}(k)
$$

Let $X^{(0)}$ be the $\mathrm{GM}(1,1)$ modeling sequence:

$$
X^{(0)}=\left(x^{(0)}(1), x^{(0)}(2), \cdots x^{(0)}(n)\right)
$$

Perform an accumulation and define a 1-AGO sequence with $X^{(1)}$ as $X^{(0)}$, then generate a sequence into the next

$$
\begin{gathered}
X^{(1)}=\left(x^{(1)}(1), x^{(1)}(2), \cdots x^{(1)}(n)\right) \\
x^{(1)}(k)=\sum_{m-1}^{k} x^{(1)}(m)
\end{gathered}
$$

The grey differential equation model of $\operatorname{GM}(1,1)$ is as follows

$$
x^{(0)}(k)+a z^{(1)}(k)=b
$$

Let $\hat{a}=(a, b)^{T}$ use the least squares method to estimate the parameters, which can be obtained:

$$
\hat{a}=\left(B^{T} B\right)^{-1} B^{T} Y_{n}
$$

among them,

$$
B=\left(\begin{array}{l}
-z^{(1)}(2) 1 \\
-z^{(1)}(3) 1 \\
\ldots \ldots \\
-z^{(1)}(n) 1
\end{array}\right), Y_{n}=\left(\begin{array}{l}
x^{(0)}(2) \\
x^{(0)}(3) \\
\ldots \ldots \\
x^{(0)}(n)
\end{array}\right)
$$

Then the time response function is as follows

$$
\begin{gathered}
\wedge(1) \\
x(k+1)=\left(x^{(1)}(0)-\frac{b}{a}\right) e^{-a k}+\frac{b}{a}, k=1,2, \cdots, n \\
\wedge(0) \\
x(k+1)={ }^{\wedge(1)} x(k+1)-{ }^{\wedge}(1)
\end{gathered}
$$

\subsection{BP Neural Network Prediction Model}

The propagation process of BP neural network is divided into two directions, namely forward propagation process and back propagation process. The forward propagation process is that the known information is the input layer, the hidden layer is the rule of limiting the value, and the output layer is obtained; the back propagation process is the calculation of the error, mainly by using the gradient descent method to adjust the weight of each neuron. There are fewer error signals.

The specific flow chart [8] is as follows

d) Establishment of GM $(1,1)$ model 


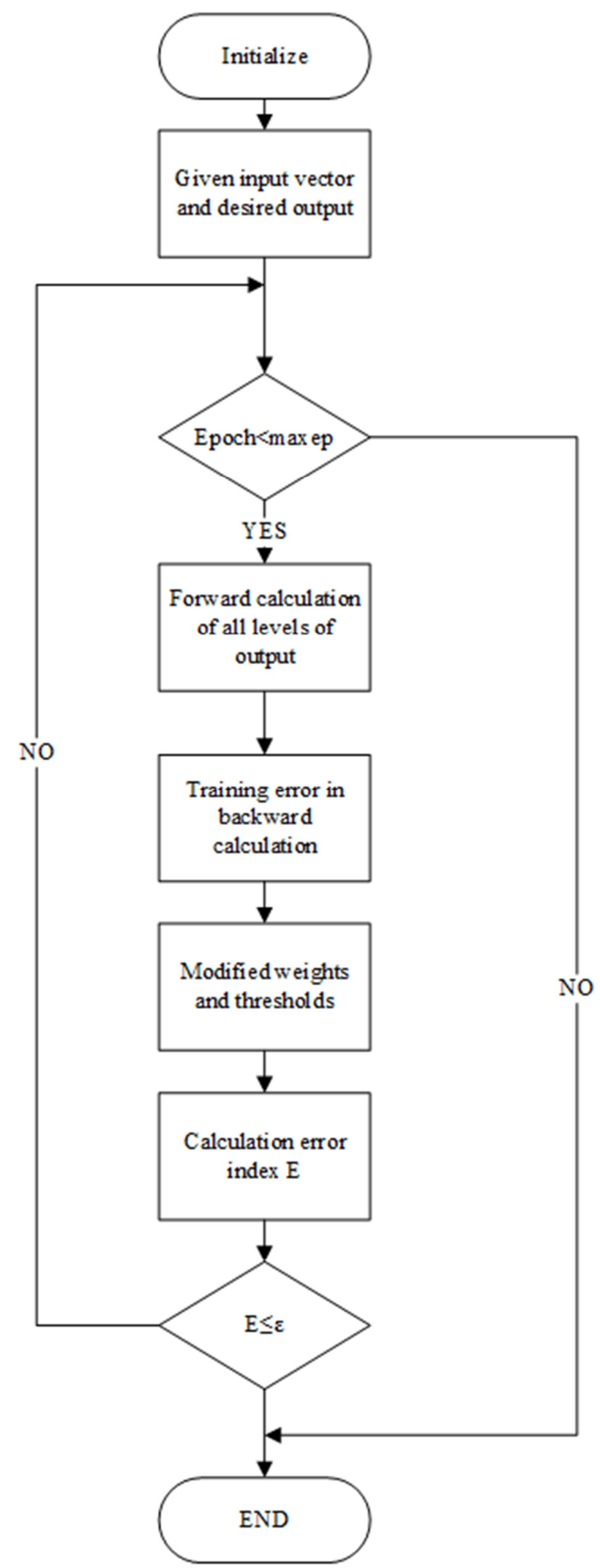

Figure 1. BP neural network flow chart.

\subsection{Grey BP Model}

The grey BP model combines the gray prediction model with the BP neural network to obtain a new prediction model containing two prediction model information, which avoids the limitations of the single model. Construct a grey BP neural network prediction model, under the specific flow chart:

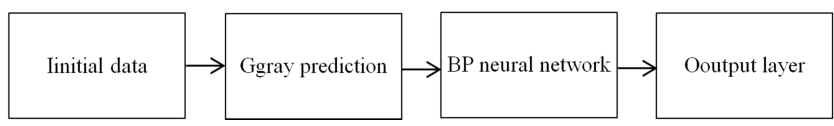

Figure 2. Schematic diagram of the gray BP correction model flow

Step 1: First, the data of 2007-2016 is used as the original sequence data, and the data is normalized by the gray prediction method.

Step 2: In the BP neural network, initialize the weight and threshold of the network, and determine parameters such as the learning rate.

Step 3: The original sequence data is divided into training data and test data (the test data does not participate in BP neural network training), wherein the training data selects the data of 2007-2014, and the test data selects the data of 2015-2016.

Step 4: Get the forecast data.

\section{Take Shandong Province of China as an Example for Analysis}

\subsection{Grey Prediction Model for Total Energy Consumption}

Based on the data from the National Bureau of Statistics' Yearbook, the grey forecasting model was established by taking the total energy consumption of Shandong Province in 2007-2016 as an example (see Table 1).

Table 1. Total energy consumption in Shandong Province from 2007 to 2016.

\begin{tabular}{ll}
\hline Years & Energy consumption (ten thousand tons) \\
\hline 2007 & 311442 \\
2008 & 320611 \\
2009 & 336126 \\
2010 & 360648 \\
2011 & 387043 \\
2012 & 402138 \\
2013 & 416913 \\
2014 & 425806 \\
2015 & 429905 \\
2016 & 435819 \\
\hline
\end{tabular}

\subsubsection{Initial Value Processing}

Get the answer: (1, 68521.01, 118794.9, 172498.4, 230284.0, 290997.8, 351980.3, 413984.2, 480518.5, $551236.8)$

\subsubsection{Forming a Gray Prediction Generation Sequence} Original series: 


$$
X^{(0)}=\left(\begin{array}{l}
43846.31,49349.41,51198.32,56208.7,59362.48,62065.18,59899.78, \\
64108.05,68960.72,72475.87
\end{array}\right)
$$

Accumulate according to (1.2) to generate a sequence:

$$
X^{(1)}=[43846.31,93195.72,144394.0,200602.7,259965.2,322030.4,381930.1,446038.2,514998.9,587474.8]
$$

\subsubsection{Construct Model Matrix and Calculate Parameters}

Calculated according to formula (11):

$$
B=\left\{\begin{array}{l}
-68521.01,1.0 \\
-118794.9,1.0 \\
-172498.4,1.0 \\
-230284.0,1.0 \\
-290997.8,1.0 \\
-351980.3,1.0 \\
-413984.2,1.0 \\
-480518.5,1.0 \\
-551236.8,1.0
\end{array}\right\} Y=\left\{\begin{array}{l}
49349.41 \\
51198.32 \\
56208.7 \\
59362.48 \\
62065.18 \\
59899.73 \\
64108.05 \\
68960.72 \\
72475.87
\end{array}\right\}
$$

Then we get the answer

$$
a=-0.04472407 b=47091.21
$$

\subsubsection{Get the Prediction Model}

Bring the above parameters into (5) and (11) to get:

$$
\begin{gathered}
\frac{d x^{(1)}}{d t}-004472407 x^{(1)}=47091.21 \\
\hat{x}^{(1)}(k+1)=-1052900+47091.2 e^{0.04472407 k}
\end{gathered}
$$

According to the formula (14), the predicted total energy consumption of Shandong Province can be obtained. The results are shown in Table 2 below;

From the data in Table 2 we can find:

a) Grey forecasting models can be used to predict the total energy consumption of Shandong Province;

b) The residual difference obtained by this model is relatively large; the persuasiveness of the model is not strong.

c) According to the relative error rate in Table 2, the average relative error from 2007 to 2016 is $7.25 \%$.

Table 2. Grey forecast value of total energy consumption in Shandong Province.

\begin{tabular}{lllll}
\hline Years & Energy consumption (10,000 tons) & Predictive value (10,000 tons) & Residual (10,000 tons) & Relative error (\%) \\
\hline 2007 & 311442 & 305449.29 & -5992.71 & $-1.96 \%$ \\
2008 & 320611 & 330122.75 & 9511.75 & $2.88 \%$ \\
2009 & 336126 & 354796.21 & 18670.21 & $5.26 \%$ \\
2010 & 360648 & 379469.68 & 18821.68 & $4.96 \%$ \\
2011 & 387043 & 404143.14 & 17100.14 & $4.23 \%$ \\
2012 & 402138 & 428816.61 & 26678.61 & $6.22 \%$ \\
2013 & 416913 & 453490.07 & 36577.07 & $8.07 \%$ \\
2014 & 425806 & 478163.54 & 52357.54 & $10.95 \%$ \\
2015 & 429905 & 502837 & 72932 & $14.5 \%$ \\
2016 & 435819 & 527510.46 & 91691.46 & $17.38 \%$ \\
\hline
\end{tabular}

\subsection{BP Neural Network Model of Energy Consumption in Shandong Province}

The specific steps are

a) Initialization: Set the initial value of each weight and threshold: $W_{j i}^{(1)}[0], \theta_{j}^{(l)}(l=0,1, \cdots, l)$ is a small random number, and this paper takes a random b) Input sample and expected output: Provide training samples and target output, 10-20 steps for each sample. The input training samples are: [43846.31, 49349.41,

\begin{tabular}{|c|c|c|c|c|}
\hline Years & Energy consumption (10,000 tons) & Predictive value (10,000 tons) & Residual (10,000 tons) & Relative error\% \\
\hline 2007 & 311442 & 349187.3 & 37745.3 & $12.12 \%$ \\
\hline 2008 & 320611 & 360266.6 & 39655.6 & $12.37 \%$ \\
\hline 2009 & 336126 & 387496 & 51370 & $15.28 \%$ \\
\hline 2010 & 360648 & 398848.8 & 38200.8 & $10.59 \%$ \\
\hline 2011 & 387043 & 404429.4 & 17386.4 & $4.49 \%$ \\
\hline 2012 & 402138 & 406014.2 & 3876.2 & $0.96 \%$ \\
\hline 2013 & 416913 & 406462.7 & -10450.3 & $-2.51 \%$ \\
\hline 2015 & 429905 & 407063.7 & -22841.3 & $-5.31 \%$ \\
\hline 2016 & 435819 & 407045.8 & -28773.2 & $-6.60 \%$ \\
\hline
\end{tabular}
51198.32, 56208.7, 59362.48, 62065.18, 59899.73, $64108.05,68960.72,72475.87]$, and the target output is limited to about $30 \%$ of the input value. The predicted values are obtained as shown in Table 3 below.

Table 3. BP neural network predicts energy results. 
c) Get the training error to meet:

$$
E<\mathcal{E}
$$

d) End of training

From the data in Table 3 you can find:

BP neural network can be used to predict the total energy consumption of Shandong Province;

The residual difference obtained by this model is smaller than that of the grey prediction model; however, there is still a problem that the model is not convincing.

According to the relative error rate in Table 3, the average relative error from 2007 to 2016 is $3.70 \%$.

\subsection{Gray BP Model}

Normalize the energy consumption data of Shandong Province from 2006 to 2017, and obtain:

$[1,68521.01,118794.9,172498.4,230284.0,290997.8$, 351980.3, 413984.2, 480518.5, 551236.8]

This paper takes the random number between trend weights $[-2,3]$, the learning rate is set to 0.001 , the target precision control parameter reached after network training is set to 0 , and the sample mode calculator and the training number calculator are set. Is 1 , error $\mathrm{E}$ is set to 0 .

Based on the revised model, the estimated energy consumption of Shandong Province in 2007-2016 using MBTLBA software is shown in Table 4 below.

Table 4. Grey BP model predicts energy results.

\begin{tabular}{llll}
\hline Years & Energy consumption (10,000 tons) & Predictive value (10,000 tons) & Residual (10,000 tons) \\
\hline 2007 & 311442 & 317702 & 6259.984 \\
2008 & 320611 & 325003.4 & 4392.371 \\
2009 & 336126 & 341436.8 & 5310.791 \\
2010 & 360648 & 364579.1 & 3931.063 \\
2011 & 387043 & 392809.9 & 5766.941 \\
2012 & 402138 & 396950.4 & -5187.58 \\
2013 & 416913 & 423125 & 6212.004 \\
2014 & 425806 & 419461.5 & -6344.51 \\
2015 & 429905 & 436310.6 & 6405.585 \\
2016 & 435819 & 435838.7 & $1.58 \%$ \\
\hline
\end{tabular}

From the data in Table 4 we can find:

The gray BP model can be used to predict the total energy consumption of Shandong Province;

The residual difference obtained by this model is small compared to the first two models; overcoming the defects of a single model

According to the relative error rate in Table 4, the average relative error from 2007 to 2016 is $2.04 \%$.

The contrast curve of the grey prediction model, BP neural network model and gray BP model relative error is obtained by using EXCEL software. The results are shown in Figure 3.

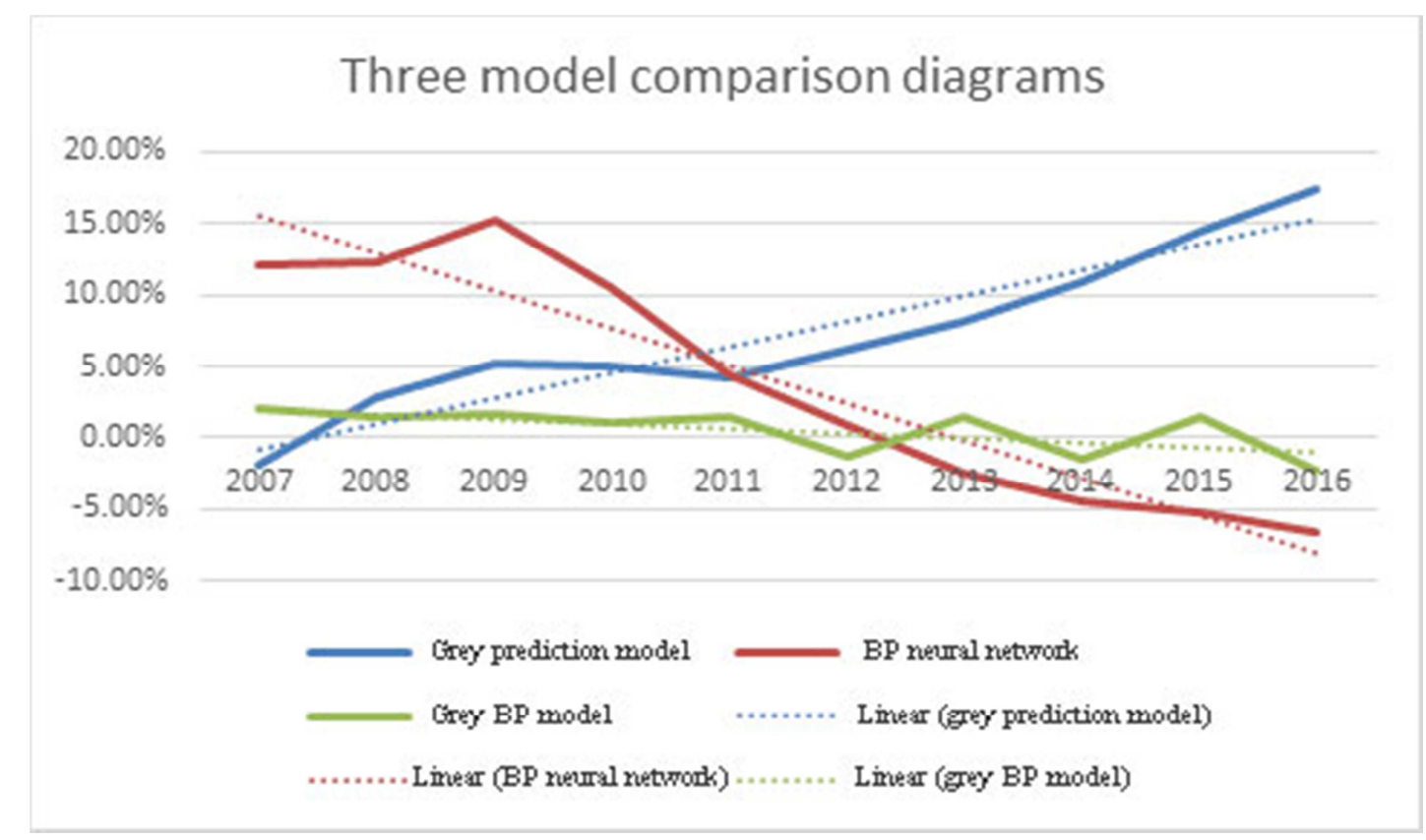

Figure 3. Comparison of three models. 
According to the trend of the fold line in Figure 3, we can get:

The change of the relative error value of the gray prediction model shows an upward trend, which indicates that the error value will become larger and larger as the number of prediction years increases, and the model becomes more and more unsuitable.

Although the relative error value of the BP neural network model shows a downward trend, the error is too large at the beginning of the prediction, and the predicted value cannot be used reliably.
The fluctuation of the relative error of the gray BP model is small and stable.

Considering the comprehensive consideration, the grey BP model is more ideal than the single prediction model in energy consumption prediction.

\subsection{Model Checking}

The test results of the three models are shown in table 5 below

Table 5. Results of three model tests.

\begin{tabular}{llllllll}
\hline \multirow{2}{*}{ Years } & \multirow{2}{*}{ Actual value } & \multicolumn{2}{l}{ Greyprediction model } & BP neural network & \multicolumn{2}{c}{ Modified model } \\
\cline { 3 - 7 } & & Predictive value & Error & Predictive value & Error & Predictive value & Error \\
\hline 2007 & 311442 & 305449.29 & $-1.96 \%$ & 349187.3 & $12.12 \%$ & 317702 & $2.01 \%$ \\
2008 & 320611 & 330122.75 & $2.88 \%$ & 360266.6 & $12.37 \%$ & 325003.4 & $1.37 \%$ \\
2009 & 336126 & 354796.21 & $5.26 \%$ & 387496 & $15.28 \%$ & 341436.8 & $1.58 \%$ \\
2010 & 360648 & 379469.68 & $4.96 \%$ & 398848.8 & $10.59 \%$ & 364579.1 & $1.09 \%$ \\
2011 & 387043 & 404143.14 & $4.23 \%$ & 404429.4 & $4.49 \%$ & 392809.9 & $1.49 \%$ \\
2012 & 402138 & 428816.61 & $6.22 \%$ & 406014.2 & $0.96 \%$ & 396950.4 & $-1.29 \%$ \\
2013 & 416913 & 453490.07 & $8.07 \%$ & 406462.7 & $-2.51 \%$ & 423125 & $1.49 \%$ \\
2014 & 425806 & 478163.54 & $10.95 \%$ & 406901.3 & $-4.44 \%$ & 419461.5 & $-1.49 \%$ \\
2015 & 429905 & 502837 & $14.5 \%$ & 407063.7 & $-5.31 \%$ & 436310.6 & $1.49 \%$ \\
2016 & 435819 & 527510.46 & $17.38 \%$ & 407045.8 & $-6.60 \%$ & 425838.7 & $-2.29 \%$ \\
\hline
\end{tabular}

By using the error rate data in the table, the average relative error of the gray BP model is calculated to be $2.04 \%$, while the gray prediction model and the BP neural network model are $7.35 \%$ and 3.70\%, respectively. This shows that the gray BP model can effectively reduce the error to achieve higher prediction accuracy.

The gray BP model is used to predict the energy consumption of Shandong Province from 2018 to 2035 . The results are shown in Figure 4 below.

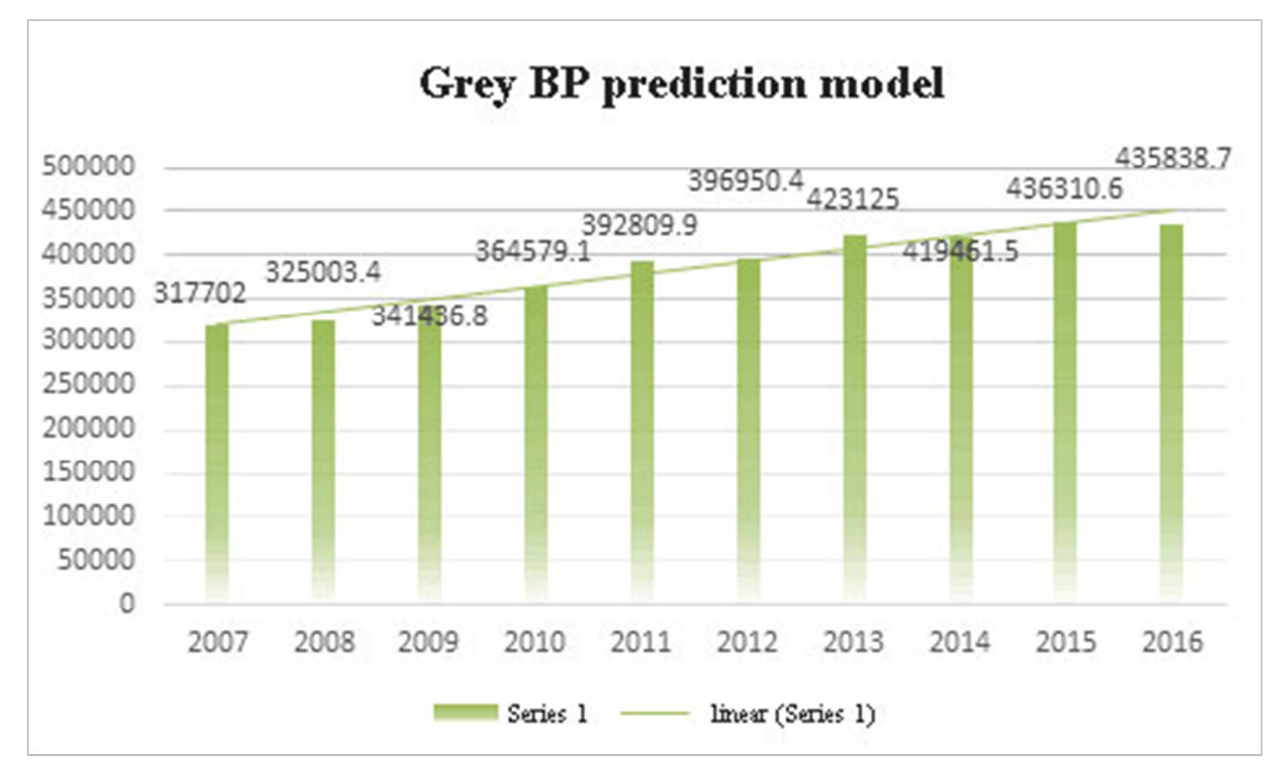

Figure 4. Forecast of energy consumption in Shandong Province from 2018 to 2035.

By analyzing the trends in Figure 4, you can get:

In general, energy consumption in Shandong Province will continue to increase from 2018 to 2035 ;

From careful analysis, it is found that energy consumption is not increasing, but a wave-like increasing trend, which may be related to accidental factors such as policy changes and climatic conditions.

\section{Conclusion}

According to the total energy consumption of Shandong Province from 2007 to 2016, the grey prediction model is used to make a preliminary prediction, and then BP neural network is used to correct it. The average error after correction is 
reduced from $7.25 \%$ to $2.04 \%$, which shows that the grey BP model is more correct. This can accurately reflect the actual situation of the total energy consumption in Shandong Province, and provide a reference for the future prediction of the total energy consumption in Shandong Province.

In the follow-up study, we can no longer be limited to the forecast of total consumption in Shandong Province. After knowing the energy consumption of other areas in the previous few years, we can put these data into the model and get the corresponding forecast value.

\section{Acknowledgements}

The authors would like to thank everyone, just everyone!

\section{References}

[1] Zhang Ming, Mu Hailin. Study on passenger transportation energy consumption in China's megacities based on LMDI decomposition method [J]. ActaScientiarum Naturalium Universitatis Pekinensis, 2010, 46 (03):483-486.

[2] Hang Chenzhe, XuDinghua, Ma Guoyuan, Zhang Haiyun, TengJunheng. Study on uncertainty evaluation method of annual energy consumption efficiency measured by air enthalpy method [J]. ActaMetrologica Sinica, 2017, 38 (01):34-39.

[3] Dai Xiaowen, He Yanqiu, ZhongQiubo. Research on driving factors and contribution of agricultural energy consumption carbon emissions in China-Based on Kaya identity expansion and LMDI index decomposition method [J]. Chinese Journal of Eco-Agriculture, 2015, 23 (11):1445-1454.

[4] Hu Bentian, Fang Chao. An empirical study on regional economic growth and energy consumption in Wanjiang City Belt_-Based on Tapio decoupling model and LMDI method [J]. Journal of Tongling University, 2014, 13 (02):68-73.

[5] Wang Yongli, Shi Dan, Han Jiqiong. Research on energy consumption in China based on multiple regression trial analysis model [J]. Management \& Technology of SME, 2014 (11):145-146.

[6] Liu Yuhai, Wu Peng. Energy consumption, carbon dioxide emissions and APEC regional economic growth-Empirical study based on SBM-Undesirable and Meta-frontier model [J]. Economic Review, 2011 (06):109-120+129.
[7] Zeng Bo, Meng Wei, Liu Sifeng, Li Chuan, Cui Jie. Grey heterogeneous data prediction modeling method for disaster emergency material demand $[\mathrm{J}]$. Chinese Journal of XuXingjun, Yan Gangfeng. Analysis of stock price trend based on BP neural network [J]. Zhejiang finance, 2011 (11):57-59+64. Management Science, 2015, 23 (08): 84-91.

[8] Wang Liping, Li Shuqin. The impact of FDI on China's low-carbon economy is based on China's data test from 1992 to 2016 [J]. Resource development and market, 1988, 34 (10):1438-1443.

[9] Zhang Jing. Prediction of PM2.5 concentration in shenyang city based on BP neural network [A]. China meteorological society. Annual meeting of the 35th China meteorological society, S12 atmospheric composition and weather, climate change and environmental impact and environmental weather forecast and impact assessment [C].

[10] Guo Xue. Grey correlation analysis of air quality influencing factors in taiyuan city [A]. China meteorological society. Annual meeting of the 35th China meteorological society, S12 atmospheric composition and weather, climate change and environmental impact and environmental weather forecast and impact assessment [C]. China meteorological society: China meteorological society, 2018:5.

[11] Decision of the standing committee of the people's congress of sichuan province to amend the "implementation measures of $>$ of energy conservation law of the People's Republic of China" [N]. Sichuan daily, 2018-10-10 (010).

[12] Zhao Wenqiang. Discussion on energy alternatives based on a low carbon background [J]. Low carbon world, 2018 (10):152-154.

[13] Tian Yingnan. Study on optimal design of distributed cold and thermal power energy system and comprehensive evaluation method of multiple indexes $[\mathrm{J}]$. Economic and trade practice, 2018 (19): 275.

[14] Ceng Qing. The price of carbon emission rights in China, the influence of two kinds of energy shares - comparative analysis based on VECM model [J/OL]. Financial development research: 1-9 [2018-10-11]. https://doi.org/10.19647/j.cnki.37-1462/f.2018.10.009.

[15] Li Yiqing, Liu Qianjin, Zhou Baorong, Liu Shiping, Cheng Lanfen. Application of power generation rights trading that takes into account carbon trading benefits in clean energy $[\mathrm{J}]$. Value engineering, 2008, 37 (31):186-189. 